\title{
A Case of Difficult Diagnosis: A Squamous Cell Carcinoma with Bone Exposure and Oro-sinus Communication in a Patient Receiving Alendronate ${ }^{\dagger}$
}

\author{
Francesca Pavanelli *, Roberto Parrulli, Giuseppe Lizio, Roberta Ippolito, \\ Salvatore Emanuele Teresi and Claudio Marchetti \\ Unit of Oral and Maxillofacial Surgery, Department of Biomedical and Neuromotor Science (DIBINEM), \\ University of Bologna, 40125 Bologna, Italy; roberto.parrulli@studio.unibo.it (R.P.); giuseppe.lizio2@unibo.it (G.L.); \\ ippolitoroberta85@gmail.com (R.I.); salvatore.teresi@studio.unibo.it (S.E.T.); claudio.marchetti@unibo.it (C.M.) \\ * Correspondence: Francesca.pavanelli@studio.unibo.it; Tel.: +39-3409668168 \\ + Presented at the XV National and III International Congress of the Italian Society of Oral Pathology and \\ Medicine (SIPMO), Bari, Italy,17-19 October 2019.
}

Published: 12 December 2019

Medication-Related Osteonecrosis of the Jaw (MRONJ) and Squamous Cell Carcinoma (SCC) are two distinct nosological entities that can affect the oral cavity, with different etiopathogenesis and histology. Nevertheless, the bone exposure surrounded by altered mucous tissue as a clinical manifestation of a therapy with anti-resorptive drugs can look similar to the clinical scenario observed in cases of oral carcinoma. Hence a correct diagnosis can be challenging [1,2].

A 71-year-old female patient, no smoker and no alcohol drinker, underwent treatment with Alendronate for osteoporosis for 4.5 years. The patient was referred by a Colleague with a preliminary diagnosis of MRONJ to the Oral and Maxillofacial Surgery Unit, University of Bologna, for the management of the symptomatic bone exposure. This scenario appeared about a month before and was preceded by swelling about two months before in the upper left molar alveolar region rehabilitated with a prosthetic bridge 10 years before. The clinical examination confirmed the presence of bone exposure in the reported site surrounded by an affected soft tissue on the palatal and buccal side: the mucosa appeared strongly inflamed with a granulomatous appearance and with rolled edges (Figure 1).

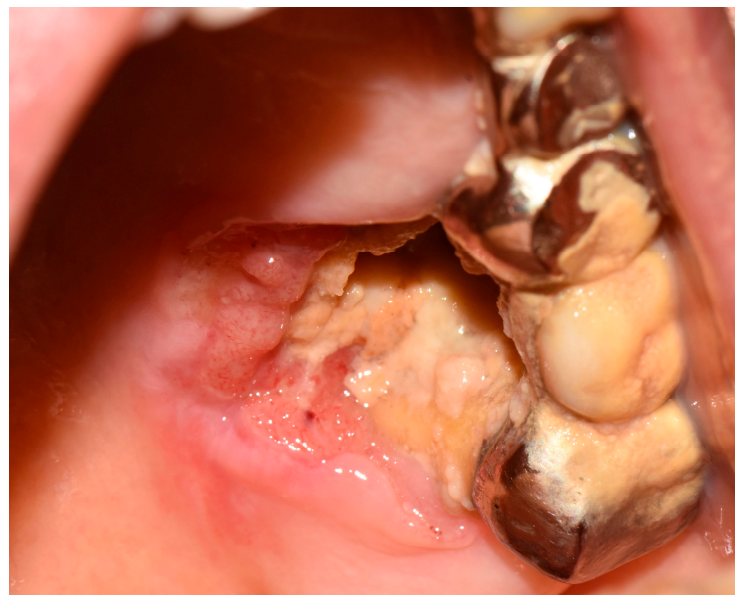

Figure 1. Clinical examination.

The panoramic x-ray did not show any sign of pathology. The Cone Beam CT revealed the presence of an oro-sinus communication of about $1 \mathrm{~cm}$ diameter, thickening of the sinus mucosa; the residual alveolar bone showed structural alterations with a chromatic radio-opaque and radio-lucent areas irregularly distributed (Figure 2). 


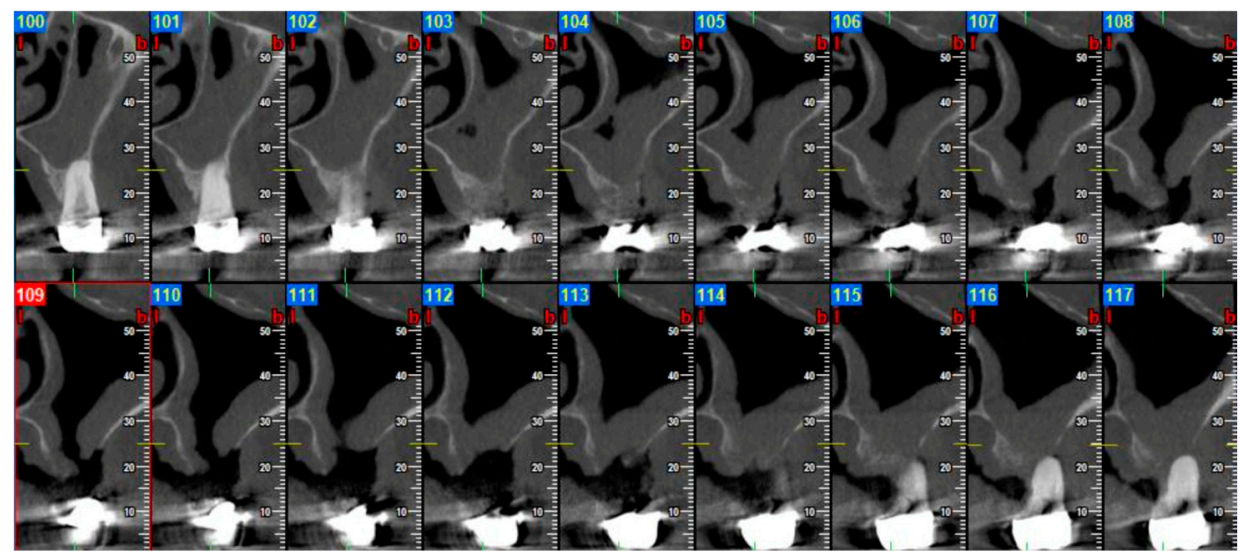

Figure 2. CBCT: paraxial cuts.

The first diagnostic option was MRONJ. Nevertheless, the particular conformation of the oral mucosa, the strong pain complained by the patient and the rapidity of occurrence usually does not match with a MRONJ scenario due to an adverse reaction to Alendronate, especially if had been assumed only for 4.5 years. The histological exam, carried out on an incisional bioptic portion of mucosa, reported the diagnosis of SCC involving the sub-mucosal layers, moderately differentiated, with signs of perineural infiltration. Hence, the patient was sent to the Maxillofacial Surgery Unit, Sant'Orsola-Malpighi Hospital of Bologna for surgical treatment in general anesthesia. We are waiting for the final histological report.

In conclusion, this singular case highlighted that the correct diagnosis is to be reached only with a careful anamnestic and clinical evaluation and a consequent peculiar diagnostic path. This goal can be reached only after a deep sharing of knowledge between the members of a specialized team that can take in consideration all the diagnostic options, even the less probable ones.

Furthermore, a question must be addressed, in our opinion, as soon as possible: which way and which relevance can the anti-resorptive drugs therapy influence the bone infiltration of an oral cavity SCC with?

Conflicts of Interest: The authors declare no conflict of interest.

\section{References}

1. Pancholi, M.; Edwards, A.; Langton, S. Bisphosphonate induced osteochemonecrosis of the jaw mimicking a tumour. Br Dent. J. 2007, 203, 87-89, doi:10.1038/bdj.2007.634

2. Tocaciu, S.; Breik, O.; Lim, B.; Angel, C.; Rutherford, N. Diagnostic dilemma between medication-related osteonecrosis and oral squamous cell carcinoma in a mandibular lytic lesion. Br. J. Oral Maxillofac Surg. 2017, 55, e53-e57, doi:10.1016/j.bjoms.2017.08.005.

(C) 2019 by the authors. Licensee MDPI, Basel, Switzerland. This article is an open access article distributed under the terms and conditions of the Creative Commons Attribution (CC BY) license (http://creativecommons.org/licenses/by/4.0/). 